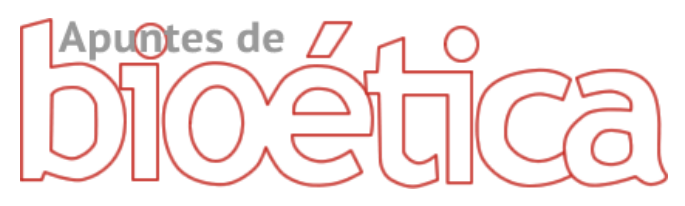

https://doi.org/10.35383/apuntes.v4i2.675 e-ISSN:2663-4910

Universidad Católica Santo Toribio de Mogrovejo

\title{
El papel de la mujer como eje clave en la historia
}

\section{The role of women as a key axis in history}

\author{
María Luisa Pro Velasco ${ }^{*}, 1$ a \\ marisa.pro@ucavila.es \\ https://orcid.org/0000-0003-1439-6328
}

\section{Rubén Blázquez Gómez ${ }^{1, ~ b}$}

ruben.blazquez@ucavila.es

https://orcid.org/0000-0003-3064-3229

\author{
*Autor corresponsal \\ ${ }^{1}$ Universidad Católica de Ávila, Ávila, España \\ a Doctorado en Filosofía \\ b Máster en Dirección de Recursos Humanos
}

Fechas importantes

Recibido: 07/10/2021

Aceptado: 21/12/2021

Publicado online: $23 / 12 / 2021$

\section{Resumen}

El papel desempeñado por la mujer en la sociedad ha ido cambiando a lo largo del tiempo. En este trabajo se estudia la historia del movimiento de liberación de la mujer, pasando por la revolución sexual, en dos momentos, para finalizar abordando el feminismo, que ha sido el gran protagonista en la lucha por mejorar la situación y que, en la actualidad, presenta diversas manifestaciones.

Palabras clave: Mujer; Liberación; Revolución Sexual; Feminismo.

\section{Abstract}

The role played by women in our society has changed over time. This article studies the history of women's liberation movement. It covers from the sexual revolution, in two different moments, to feminism, which has been the major player in the struggle to improve the situation, and it currently presents various expressions.

Keywords: Women; Liberation; Sexual Revolution; Feminism. 


\section{Introducción}

En un mundo cada vez más globalizado, el problema del papel de la mujer ha llegado a ser una cuestión apremiante, que no solo preocupa al lado femenino de la humanidad, sino que, por su envergadura, se trata de un problema social. El papel que ha de desempeñar la mujer en el siglo XXI, así como la compatibilidad entre la vida personal y la vida profesional son solo algunas de las líneas que habría que esbozar con mayor precisión. Pero no solo eso, también la familia y la sociedad se han visto afectadas por esta problemática, porque durante siglos, la mujer ha sido el corazón de la familia y, a través de ella ha realizado una importantísima aportación a la sociedad. Hoy en día, la mujer ha asumido nuevas funciones y papeles que, en ocasiones, le hacen dejar en un segundo plano lo que ha sido -mucho más que una opción- su esencia: la maternidad. Como dice Sara Gallardo, directora de la Cátedra "Santa Teresa de Jesús" de estudios sobre la mujer, de la Universidad Católica de Ávila en una de sus publicaciones: "En nuestra feroz sociedad, que una persona normal llegue a «ser alguien» implica pagar el alto precio de renunciar a dedicarse a las demás cosas que hacen de una vida humana, precisamente, una vida «normal»" (Gallardo, 2017, p. 195). Esto es precisamente lo que le está pasando en numerosas ocasiones a la mujer de nuestro siglo.

En este trabajo se trata de entender el movimiento de liberación de la mujer a lo largo de la historia. Se examina igualmente, dividido en dos momentos, el fenómeno de la revolución sexual (Kuby, 2017), para entender su relevancia social. Y se trata sobre el feminismo y sus distintos tipos o manifestaciones actuales, con el objetivo de asumir y difundir un planteamiento que lleve a la verdadera realización de la mujer. Finalmente, se sacan algunas conclusiones de lo expuesto.

\section{Historia del movimiento de liberación de la mujer}

Una de las cuestiones más relevantes y debatidas de nuestro siglo es la del papel de la mujer. Ciertamente, cada vez podemos observar en más ocasiones cómo más mujeres acceden a puestos que nunca antes habían podido ocupar. Ahora bien, este siglo de la revolución digital, las comunicaciones sociales y los avances científico-técnicos, lo es también de las fake news. Por ello, en este apartado, pretendemos dilucidar hechos importantes desde la Antigüedad hasta nuestros días, pasando por la Edad Media, el Renacimiento y la Modernidad. 
Comencemos por la Antigüedad griega, puesto que la mujer apenas ocupaba en Grecia papel social ninguno, raras y escasas son las mujeres con cierto protagonismo o relevancia social, cuyos nombres hayan llegado hasta nuestros días. Podemos pensar quizá en los nombres de Jantipa, la esposa de Sócrates, o de la de Aristóteles, Pitias de Aso, sabiendo que no jugaron sino un papel muy secundario en las obras de estos conocidos pensadores. También podrían mencionarse: la poetisa Safo de Mitilene, Aspasia de Mileto o Diotima.

Por lo que se refiere al cristianismo, no cabe la menor duda de que su iniciador, Cristo mismo, supuso un punto de inflexión en la historia de la humanidad, $y$, con él, también los seguidores de su doctrina, aunque en ocasiones este avance ha sido lento y progresivo a lo largo de la historia. En la actualidad hay una profunda ignorancia hacia todo lo que tenga que ver con la religión (a pesar de ser esta una constante antropológica). No podemos dejar de advertir el trato ciertamente novedoso de Jesús de Nazaret con algunas mujeres de su tiempo. El primero de ellos, y más llamativo quizá, a pesar de ser una cuestión de fe, es su nacimiento sin intervención de varón (LC 1, 26-38). Posteriormente, por mencionar solo algunos ejemplos, podemos pensar en el pasaje de Jesús con la mujer samaritana junto al pozo de Jacob (Jn 4, 5-43): mientras los discípulos fueron a comprar provisiones, al volver lo encontraron hablando con una mujer samaritana. Algo poco común para un hombre judío, no solo por el hecho de hablar con una mujer, sino además porque los samaritanos eran considerados alejados de la verdadera tradición, por tanto, infieles.

Otro ejemplo puede ser el diálogo de Jesús con la pecadora pública en casa del fariseo (Lc 7, 36-50), o con la mujer descubierta en adulterio, a la que defiende ante los hombres del pueblo que pretendían lapidarla viva (Jn 8, 1-11), costumbre que, lamentablemente, sigue vigente en países de credo musulmán, especialmente en África y en Asia. Y, asimismo, es llamativa la amistad y la confianza de Jesús con Marta y con María, hermanas de Lázaro, y sus visitas a Betania (Jn 12, 1-3). Recordemos cómo Marta logró de Jesús el milagro de la resurrección de su hermano (Jn 11). Finalmente, cabe destacar el papel de las mujeres que le seguían y que, por tanto, le acompañaron además de sus discípulos durante el tiempo de su vida pública (María Magdalena, María la madre de Santiago y de José, y la madre de los hijos de Zebedeo...) (Mt 27, 55-56). Y no solo eso, sino que, al pie de la cruz, fueron algunas de ellas sus más fieles seguidoras (Jn 19, 25), a diferencia de los discípulos que, a excepción de Juan, no estuvieron en el Calvario (Jn 19, 25-27). Luego, tras hacer mención de estos pasajes evangélicos, es llamativo que, no solo a la mujer, sino también a los niños y esclavos se les respetase más a partir de la extensión del cristianismo. 
Podemos pensar también acerca de cómo el matrimonio cristiano supuso también una mejora de la situación de la mujer en la familia y en la sociedad, pues exigía un cierto respeto (Bel Bravo, 1998 y 2020).

En cuanto a la Edad Media, a pesar de que se cuenta que fue un período oscurantista, quizá en él hubo más luces que en la Ilustración por lo que al movimiento de liberación de la mujer se refiere.

Por un lado, a partir de esta época y, a partir del Renacimiento, empezamos a conocer nombres de mujeres relevantes en la historia de la humanidad en mayor número que en la Antigüedad. Este es un hecho que no puede pasar inadvertido, y que, por tanto, le invita a preguntarse por su causa. ¿No trabajó acaso la mujer igual que cualquier otro hombre en gremios, y aprendía el oficio familiar? ¿No permitió la vida retirada en un convento, entre otras cosas, dedicarse al estudio? ¿No era la mujer acaso en estas épocas -y sigue siendo- el corazón del hogar y de la sociedad?

Si queremos descubrir el papel de la mujer en la sociedad a partir de la Edad Media, por un lado, podemos pensar en la relevancia social que tuvieron los monasterios y abadías femeninas como reductos de conservación de la cultura (Bel Bravo, 2020). En esta atmósfera surgieron algunas de las primeras filósofas, teólogas e incluso farmacéuticas, gracias a los conocimientos que pudieron desarrollar en este contexto. Pensamos, por ejemplo, en Hildegarda de Bingen. Asimismo, recordemos que en este periodo histórico el poder religioso estaba unido al poder político. Por tanto, con facilidad nos podemos hacer cargo del papel que podía tener una abadesa en la sociedad de su tiempo: al cargo de toda una comunidad de mujeres.

Por otra parte, con el descubrimiento de América, no fueron pocos los adelantos en la mejora del trato hacia mujeres y niños. Así, llama la atención que, tras el descubrimiento de América y la conversión de los primeros indígenas a la fe, algunas mujeres conversas no querían casarse con los no conversos, pues suponían que serían menos respetadas por ellos que por los hombres cristianos, que evitaban la poligamia y el incesto, entre otras prácticas (Cantera Montenegro, 2019).

Se implantó una cierta regulación del trabajo de las mujeres, especialmente de las embarazadas y de los infantes (Inaraja, 2012 e Inaraja y Expósito, 2015). Por otro lado, también hay que hacer hincapié en la labor caritativa de la Iglesia en no sólo de enseñanza 
elemental para hijos de caciques, sino también de enseñanza de la mujer y enseñanza elemental para niños (Borges Morán, 1992).

Asimismo, si regresamos a Europa, ya en el Renacimiento, los conventos eran, en muchas ocasiones, lugares de verdadera promoción y formación de la mujer a nivel integral, como lo fue el Monasterio de Nuestra Señora de Gracia en Ávila para quien sería conocida después como Teresa de Jesús, de quien lleva el nombre la Cátedra de Estudios sobre la mujer de la Universidad Católica de Ávila. Al morir su madre, fue llevada por su padre al internado que tenían las Madres Agustinas En este convento se formó y, aunque entró "enemiguísima de ser monja", se le iría yendo el miedo animada por María de Briceño, su formadora (Teresa de Jesús, 2014, 41). Resulta difícil imaginar que Teresa llegase a ser quien fue sin su paso por este lugar de educación y formación integral, al faltarle su madre y llevarse varios años con sus hermanas mayores y menores.

Por otro lado, como segundo acontecimiento importante en el Renacimiento, sabemos que algunas mujeres pudieron acceder a la Universidad a partir del siglo XVI. Pensemos en la Universidad de Salamanca, a donde podían acudir a formarse las hijas de nobles y burgueses, incrementando así su relevancia social (Bel Bravo, 2020). Ejemplos de mujeres cultas y de cierto renombre que han llegado hasta nuestros días y que se formaron en dicha universidad son, por ejemplo, Beatriz Galindo, más conocida como la Latina, por sus altos conocimientos del latín, de quien llevan el nombre calles, barrios y centros españoles. Así como de Lucía o Luisa de Medrano, que en la ciudad de Salamanca cuenta con centro de educación secundaria con su nombre. Ellas fueron algunas de las primeras estudiosas en este núcleo cultural. El problema surgió cuando las puertas de las universidades, no acogieron a más mujeres durante trescientos años. Ahora bien, como siempre que se restringe el acceso a algún lugar, hay cierta picaresca para saltar por encima de la norma impuesta. En el caso que nos ocupa, no faltaron mujeres que, disfrazadas de hombre, siguieran asistiendo y formándose en la universidad.

Por otra parte, al comienzo de la Modernidad, a pesar de ser el Siglo de las Luces y del aparente triunfo de los principios de la razón, se da un retroceso notable en la importancia del papel de la mujer, pues sus opciones de vida quedan reducidas socialmente, y sin posibilidades de elegir. A este respecto, se afirma: "[...] a diferencia de lo que había sucedido en la Edad Media, las mujeres en la época moderna fueron excluidas de la participación en la vida política, económica y cultural" (Bel Bravo, 2020, p. 70). Y la causa la tenemos, según esta misma autora en "la progresiva influencia del derecho romano, los 
principios de la Modernidad y el Código Napoleónico de 1804" (Bel Bravo, 1998, p. 47). Y así, la vida de la mayor parte de las mujeres se mantuvo dentro de los esquemas de la subordinación en un segundo plano.

Si nos acercamos más a lo que ha sido el movimiento de liberación de la mujer en nuestro tiempo, podemos advertir que son tres los grandes hitos por los que ha tenido y todavía hoy tiene que luchar: el derecho al voto, primero, la alfabetización y la educación superior, después, y, finalmente, el reconocimiento en el lugar de trabajo, con el respeto a su peculiar condición femenina, siendo este último, quizá, el que más nos debería preocupar en Occidente en la actualidad.

Comencemos por el primero de estos tres ejes en torno a los cuales se vertebra el feminismo: el derecho al voto. Es en el siglo XIX cuando las mujeres van a comenzar a unirse de manera más organizada para luchar por la emancipación de su sexo en los diversos movimientos feministas europeos. Así, poco a poco, las sufragistas fueron consiguiendo el perseguido derecho al voto que, hasta entonces era un privilegio únicamente masculino. Un ejemplo quizá no muy conocido de una mujer que luchó por el mismo es el de la filósofa judía alemana Edith Stein:

[...] cuando Edith [Stein] era estudiante universitaria (1911-1916) no existía el derecho de voto de la mujer, que no sería reconocido hasta la constitución de 1919, durante la República de Weimar. De hecho, ella perteneció a la asociación prusiana que luchaba por este derecho (Ramos Gómez, 2017, 70).

En definitiva, el sufragismo puede ser considerado como el primer escenario del movimiento a favor de los derechos de la mujer.

En cuanto al segundo de los hitos, la doctora Bel Bravo trae a colación algunas de las promotoras e iniciadoras de la búsqueda de formación integral para la mujer. En efecto, con la educación integral de las mujeres, es mucho lo que está en juego pues, la mayor adquisición de conocimientos y de experiencia de vida, no solo redunda en su favor, sino en el de toda la familia, por consiguiente, en el de toda la sociedad, pues esta no es nada sin todas las familias que la componen.

Finalmente está la cuestión de la mujer y su relación con el trabajo. En el momento de abordarla planteamos algunas reflexiones al respecto. Una de ellas, siguiendo a Lydia Jiménez, presidenta del Consejo Directivo de la Universidad Católica de Ávila, quien dice:

Asistimos en nuestra sociedad a un cambio social muy marcado por la mujer y su papel en todos los ámbitos: desde el siglo XVIII los esfuerzos reivindicativos han 
introducido la idea -masculina- de que la dimensión pública de la persona es requisito imprescindible para un reconocimiento justo de su valor, y de que, en este sentido, la mujer debe conseguir hacerse presente en muchos campos y ocupaciones hasta entonces impensables (Jiménez, 2017, 11).

Ciertamente, hemos de plantearnos si esto es necesariamente así, es decir, ¿solo podemos considerar como trabajo al que lo es fuera de casa y exclusivamente si este es remunerado? ¿Tiene algún valor el que tantas personas, fundamentalmente mujeres, hayan dedicado la mayor parte de sus vidas a la crianza y educación de sus hijos y a cuidar de los más débiles y enfermos de la familia?

A comienzos del siglo XXI vemos cómo muchas mujeres dan prioridad a sus carreras profesionales por encima de su vocación personal que, puede ser, aunque no únicamente, al matrimonio y, por consiguiente, a tener y a atender a unos hijos en colaboración con su marido. Esta es una opción totalmente legítima. Ahora bien, la cuestión que sería conveniente plantear aquí es la de si cabe la posibilidad de que alguna mujer opte por esta segunda opción del matrimonio y la maternidad como formas de llevar una vida plena, o solo por la primera opción, la promoción profesional. En realidad, ni hay ayudas suficientes, ni medidas públicas, ni está bien visto socialmente dedicarse a la segunda, al menos en Europa.

Por otro lado, cabe destacar también que se ha producido un importante desajuste social, derivado del acceso de la mujer tanto al poder político como social. Precisamente, porque:

[...] si bien las mujeres están ocupando lugares a los que antes se veían imposibilitadas de acceder por distintos motivos, también es verdad que los trabajos desempeñados en otras épocas por ellas ahora adolecen de atención y cuidado, creándose, por tanto, un importante desajuste social (Bel Bravo, 1998, 7 y 2020, 11).

Si las mujeres no se ocupan de aquello que solían hacer tradicionalmente, ¿quién lo hará?, ¿quién estará dispuesto a ser su sustituto?

\section{La revolución sexual}

Es así como llegamos hasta la llamada primera revolución sexual, que tuvo su auge entre los años 60 y 70 del siglo XX, tanto en Norteamérica como en Europa, con Mayo del 68 como máximo exponente (Jiménez, 2020). A este respecto, traemos a colación una reflexión de la doctora Lourdes Redondo: 
Agentes directos o indirectos, estos y otros factores causan la primera revolución sexual y un feminismo entendido, no como reivindicador de derechos legítimos de las mujeres, que también se había dado desde finales del XIX, sino de una libertad absoluta, desligada de toda traba moral y del "rol opresor" de la maternidad. De este modo, la "igualdad" se conseguiría mediante el "amor libre" -sexo libre, en realidad, la destrucción del matrimonio y de la familia tradicionales, la separación del sexo de la maternidad, posible gracias a la píldora anticonceptiva y al aborto. Estas eran las ideas de Simone de Beauvoir y de la revolución del 68. Empezaron reclamando igualdad de derechos, como otros movimientos feministas; pero, creyendo que la culpa la tenía la "diferencia" sexual, decidieron acabar con ella negando la naturaleza femenina - "no naces mujer, te hacen", la conocida frase de Simone de Beauvoir-, la maternidad y el matrimonio (Redondo, 2016, 215-216).

Es decir, que el feminismo, que parecía estar llegando a buen puerto al conseguir la igualdad de condiciones, el derecho al voto, la enseñanza y la alfabetización femeninos, así como el acceso y cierto reconocimiento en el lugar de trabajo, empezó a dar un giro y a distanciarse de este ideal. La libertad pasó a ser entendida como libertad absoluta de opciones y posibilidades. La igualdad, por su parte, se entendió como masculinización de la mujer, haciéndola renunciar al don de la maternidad y de la entrega en el matrimonio. Esto fue posible mediante el sexo libre, la píldora anticonceptiva y el aborto, que desligaron la unión sexual de la posibilidad procreativa. En relación con este planteamiento de la primera revolución sexual, cabría plantearse si realmente ayudó a la mujer a liberarse y, por consiguiente, a sentirse más realizada y plena.

Hay quienes invitan a realizar una reflexión más detenida sobre este hecho, apuntando a algunas de sus consecuencias, como pueden ser la separación de cuerpo y persona, la separación de la sexualidad y del amor y la separación de la sexualidad y de la procreación (Gallardo Martín, A. I., y Sánchez Martínez, M., 2016). Y, por lo tanto, podemos apreciar en el aumento de divorcios, parejas poco estables, madres y padres solteros, etc., sobrevenidos consiguientemente por esta primera revolución (Redondo, 2016).

Por otra parte, hay quien ya ha hablado de una segunda revolución sexual, acontecida a causa de la ideología de género. En línea con el planteamiento anterior, se sigue el proyecto marxista de la lucha de clases, aplicado a las relaciones entre el hombre y la mujer, siendo la mujer la explotada, y el varón el explotador. El lugar de la explotación en esta ocasión, no sería la fábrica, sino la familia, el propio hogar. Y el instrumento de explotación sería la maternidad (Redondo, 2016). En relación con esta idea, afirma Amaya Azcona, directora General de Red Madre en España, que: "La maternidad es percibida como un freno al desarrollo personal y profesional de la mujer" (Azcona, 2018, p. 88). Y este sería 
precisamente uno de los mayores problemas que está trayendo consigo esta ideología para la sociedad del siglo XXI. ¿Verdaderamente debe la mujer optar solamente por su carrera profesional? ¿No le es lícito hacer carrera también en el ámbito personal? Es cierto que no podemos pensar en una vuelta a sociedades anteriores a la del silgo XIX, con los derechos que se han ido logrando. Pero tampoco podemos quedarnos meramente pensando en que en el siglo XXI ya no se puede realizar ninguna aportación más a la sociedad de nuestro tiempo. Antes bien, quizá sea el momento de empezarnos a plantear cómo se puede realizar un reparto más equitativo de los papeles de hombres y de mujeres, no solo en las tareas domésticas, sino también en las profesionales. Asimismo, habría que tomar conciencia de qué podemos reivindicar al Estado como ciudadanos activos, siendo de gran importancia la implantación de medidas que ayuden a fomentar correctamente la paternidad y la maternidad de manera responsable. De hecho, hoy en día no existen posibilidades reales de conciliación, que permitan armonizar la familia con el trabajo durante los meses iniciales de la vida del hijo, o durante un tiempo más o menos prolongado. Esto nos hace ver que la familia, a pesar de ser la célula de la sociedad, no recibe la atención necesaria.

Ciertamente, de trabajar o no trabajar no se sigue una mayor realización de la persona y, concretamente, de la mujer, que es el tema que nos ocupa. En efecto, a la doble jornada laboral puede añadírsele la mala conciencia y el sentimiento continuado de la mujer por estar haciendo algo mal que, en realidad, viene derivado de no poderse entregar plenamente a una o a otra tarea.

Ciertamente, todas estas son cuestiones que requieren un análisis detenido y un examen no solo individual, sino también social. Por nuestra parte, si observamos más de cerca los fundamentos sobre los que se apoya la ideología de género, podremos advertir que está centrada en una visión subjetivista de la realidad. Al afirmar que no existe una identidad sexual, sino solamente géneros (Burgraff, 2007), en tanto que construcciones culturales, que elige cada cual, se está cayendo una vez más en la vieja máxima de Protágoras: "el hombre es la medida de todas las cosas". Esta interpretación se identificaría, por su parte, con un relativismo antropológico que, por la importancia que tiene a nivel social y, casi podría ser entendido como un relativismo cultural. Ahora bien, ¿es el hombre solo cultura o tiene también una naturaleza?

La ideología imperante hoy nos hace creer que estos dos elementos se oponen diametralmente, y que todo en el hombre es debido a la cultura. Pero, ¿qué ha sido de la naturaleza del hombre?, ¿qué podemos decir aún de su biología? ¿no tienen acaso relevancia 
para el comportamiento de la persona el ser XX o el ser XY? (Redondo, 2016). Deducimos, por tanto, que, en cuanto a su base, la ideología de género es contradictoria. Por un lado, por estar asentada sobre el relativismo que, a nivel lógico, incumple el principio de no contradicción. Por otro, por su oposición entre cuerpo y espíritu, materia y alma, o naturaleza y cultura, tratando de exaltar la vertiente cultural y su importancia para la persona, tratando de olvidar la naturaleza humana sin la cual, el hombre no puede desarrollar ese plus de racionalidad que es la cultura. Sobre esta cuestión de la naturaleza humana afirmaba Spaemann:

La estructura fundamental de esta idea es que la naturaleza produce en el hombre algo que es más que naturaleza ("nobilior", lo llama santo Tomás). El hombre no es este más; es el ser en que la naturaleza se supera a sí misma en dirección a este más (Spaemann, 1989, p. 41).

Cabe recordar que, hasta el momento, no conocemos ningún otro animal, aparte del ser humano, que tenga cultura. A continuación, veamos las diversas manifestaciones de ese movimiento social y cultural que ha sido el feminismo a lo largo de la historia, y la tipología más característica en la actualidad.

\section{El feminismo y sus manifestaciones en la actualidad}

En primer lugar, el feminismo es definido como un principio o movimiento que persigue la igualdad de derechos entre el hombre y la mujer ( $R A E, 2020$ ). Como principio teórico esencial defiende que hombres y mujeres tengan los mismos derechos, $y$, como movimiento lo que persigue es la realización efectiva de este principio. Sin embargo, a lo largo de la historia se ha creado mucha controversia sobre la finalidad real de este movimiento por parte de algunos sectores, confundiendo desde su base, el perseguir la igualdad de derechos con la igualdad entre hombres y mujeres, olvidando las propias diferencias biológicas. Al igual que el papel de la mujer ha ido cambiando a lo largo de la historia -como hemos visto en el apartado anterior-, el término feminismo también ha ido adquiriendo diferentes connotaciones con el paso de los años. Estos matices son los que nos servirán para la enunciación que realizaremos a continuación. A pesar, de poder existir varios tipos de feminismo, se produce una lucha por parte de algún movimiento, por apropiarse del término en su más amplio sentido, para la lucha por su causa.

Aunque muchos escritos sitúan el comienzo del movimiento feminista décadas después, en el siglo XVIII se produjeron las primeras manifestaciones públicas de las mujeres por la lucha 
de sus derechos, se originó un fuerte protagonismo por su parte en los hechos revolucionarios. En estos años, se iniciaron marchas armadas de mujeres en Francia cuya pretensión era conseguir no ser excluidas de la vida política y alcanzar una educación igualitaria para hombres y mujeres, sin embargo, sus peticiones fueron rechazadas. Con la Revolución Francesa, se motivó la Declaración de los Derechos del Hombre y del Ciudadano de 1789, este hecho provocó el malestar de muchas mujeres que vieron cómo fueron excluidas de esta declaración.

Cabe destacar la labor de la escritora francesa Olympe de Gouges (1748-1793). Ella replicó el texto "Historia del movimiento de liberación de la mujer", ella replicó el texto de la Declaración escribiendo la Declaración de los Derechos de la Mujer y de la Ciudadana en 1791, reivindicando todos los derechos civiles para la mujer, se convirtió en uno de los primeros textos que propugnó la igualdad legal y jurídica de las mujeres. Por su parte, la escritora y filósofa inglesa Mary Wollstonecraft (1759-1797), también desempeñó un papel importante a través de su Vindicación de los Derechos de la Mujer, texto que con el tiempo ha sido considerado por muchos como el fundador del feminismo. Con esta primera ola del feminismo el objetivo era evitar la desigualdad tanto en la educación como en la jerarquía de derechos entre hombres y mujeres. La reivindicación dio sus frutos y años después las mujeres fueron incluidas en la Declaración de los Derechos Humanos. Este logro conllevó que se iniciase una corriente de pensamiento que consideraba que las diferencias entre hombres y mujeres no eran naturales, como se pensaba hasta ese momento, sino culturales. Por lo que una educación igualitaria haría pareja a ambos sexos. El fruto principal de estos años fue que el problema de la mujer pasó de tratarse de una queja individual a un problema colectivo (Aguiar Barriga, 2020).

Estos movimientos no fueron bien vistos y se inició una persecución y represión hacia ellos. De este modo, se prohibió la aparición de mujeres en cualquier tipo de actividad política e incluso algunas de sus líderes fueron encarceladas y ejecutadas, como fue el caso de Olympe de Gouges, guillotinada en 1793. A principios del siglo XIX el Código Civil Napoleónico de 1804 se extendió por toda Europa y, a través de él se negaban todos los derechos conseguidos por la mujer durante los años anteriores, además de dictaminar una serie de leyes discriminatorias, se consideraba que las mujeres debían prestar obediencia a sus maridos, y que por naturaleza debían estar excluidas del derecho de ciudadanía. Asimismo, el hogar pasó a ser definido como el ámbito exclusivo de la actuación femenina. Además, se prohibieron las reuniones de más de cinco mujeres (De Miguel, s. f.). 
A pesar de la represión, muchas mujeres siguieron organizándose de forma clandestina. En la segunda mitad del siglo XIX y principios del siglo XX, surgió la denominada Segunda ola del feminismo. Esta segunda ola se inició principalmente en Estados Unidos, Reino Unido y algunos países de América Latina, aunque poco a poco fueron influyendo en el resto de países. El feminismo empezó a convertirse en un movimiento social de carácter internacional. Uno de los principales objetivos que se persiguieron fue el derecho al voto, de ahí que esta segunda ola también sea conocida como sufragismo. A diferencia de la primera ola, donde se produjo un movimiento más intelectual, esta segunda ola se caracterizaría por ser un movimiento de acción social.

En Estados Unidos, el movimiento feminista estuvo inicialmente relacionado con el movimiento abolicionista, ya que años atrás hubo mujeres que unieron sus fuerzas en la lucha contra la esclavitud. En 1848 se aprobó en Nueva York la Declaración de Seneca Falls, también conocida como la Declaración de Sentimientos, en la que apelaban a una única ley natural que consideraba que los hombres y mujeres son creados de la misma manera y deben tener acceso a los mismos derechos. En este sentido, podemos destacar la labor de las activistas Lucretia Mott (1793-1880) y Elizabeth Cady Stanton (1815-1902) (Miyares, 1999). En estos momentos la lucha se centraba en conseguir derechos civiles iguales a los del hombre como la igualdad de educación, así como acceder al sufragio universal. Consideraban que alcanzando el derecho a voto tendrían más fácil ir consiguiendo el resto de propósitos. Además de mediante escritos y publicaciones, las mujeres comenzaron a defender sus derechos en masa, y de este modo es como se empezaron a producir las primeras manifestaciones feministas. Finalmente, en 1920, lograron el sufragio femenino.

En Europa, el movimiento sufragista más importante se produjo en Reino Unido, el sufragio se consiguió en 1918, pero con muchas limitaciones, y no fue hasta diez años después cuando las mujeres consiguieron este derecho en igualdad de condiciones que los hombres. La lucha en Reino Unido no fue tan moderada como en Estados Unidos, sino que se produjeron actos más radicales como huelgas de hambre, encadenamientos e incluso ataques o sabotajes a dirigentes políticos. Parte de las responsables de estos actos fueron las suffragettes, que reivindicaban el derecho de las mujeres, aunque para ello tuviesen que pasar por encima de la ley. Al llegar a su fin la primera Guerra Mundial, las mujeres fueron consiguiendo el derecho al voto en otros países del mundo. En el caso de España llegaría finalmente en 1931 con la Segunda República. De las grandes potencias europeas, 
solamente Francia e Italia se quedaron atrás, consiguiendo el sufragio femenino en 1944 y 1945, respectivamente.

Por otra parte, podemos situar el origen del Feminismo Socialista en Hispanoamérica. En efecto, la escritora y pensadora francesa, Flora Tristán (1803-1844) es considerada como una de las precursoras de este feminismo. Ella consideraba que las mujeres sufrían una doble discriminación: de clase y de género, definiendo a la mujer como la proletaria del proletariado. Flora Tristán a lo largo de todo un capítulo de su obra Unión Obrera se encargó de explicar cuál era la situación en la que se encontraban las mujeres (De Miguel, s. f.). El feminismo socialista supuso que por primera vez se empezasen a dilucidar dos ramas o corrientes del feminismo. Por un lado, aquel que busca la igualdad de derechos, y, por otro lado, el que busca acabar con el sistema para conseguir sus objetivos. El feminismo socialista sería el que considera que la mujer se encuentra oprimida por el sistema capitalista y el patriarcado y, por tanto, la única manera de acabar con esta opresión consistiría en romper con estas barreras económicas y culturales que sitúan a la mujer en desventaja.

Tras la Primera Guerra Mundial, la mujer empezó a ocupar en las fábricas muchos puestos anteriormente reservados a los hombres. En estos momentos, los países más industrializados reconocían el sufragio femenino, y la lucha de la mujer se centró en conseguir los mismos derechos que los hombres en los puestos de trabajo. Sin embargo, durante el periodo que va desde la Primera Guerra Mundial hasta el fin de la Segunda Guerra Mundial, el movimiento feminista estuvo sumido en una especie de letargo. Las mujeres habían conseguido el derecho al voto y podían acceder a la universidad, lo que provocó que muchas de las mujeres, una vez alcanzados sus objetivos, se desmovilizasen. Las demandas de las décadas anteriores habían sido satisfechas y una calma aparente parecía reinar en la mayoría de los hogares. Sin embargo, se trataba de una tranquilidad momentánea, pues pronto los movimientos feministas comenzarían a florecer de nuevo.

A mediados del siglo XX, en el contexto del mayo francés (Aparisi Miralles, 2020), apareció una de las figuras más importantes del feminismo, la escritora y feminista francesa Simone de Beauvoir (1908-1986). En 1949 publicó la obra El Segundo Sexo (De Beauvoir, 2013), que es considerada una de las obras fundamentales de la historia del feminismo. Con esta obra la autora consiguió remover las conciencias y sirvió de impulso para que los movimientos feministas se reactivasen al menos en una cierta dirección de izquierdas. En esta obra, Simone de Beauvoir, trató de explicar cómo el concepto de mujer de la época era el fruto de una construcción social y cultural. Haciendo una crítica al papel asumido por la mujer a lo 
largo de la historia, las animaba a recuperar su propia identidad desde sus propios criterios. Ella sostuvo que la mujer generalmente no es definida por su propio sexo biológico, sino por una serie de roles con los que debe cumplir para ser considerada mujer, como son el ser esposa, madre, ama de casa, etc. A partir de esta obra, aunque no solamente, comenzó a distinguirse entre los conceptos de sexo y género. La autora sostenía la idea de que no se nace mujer, sino que se llega a serlo. La escritora en su obra criticaba también el androcentrismo, que situaba al hombre como la norma, lo que provocaba que la mujer no pudiera identificarse a sí misma como sujeto, sino más bien por lo que el hombre y la sociedad esperan de ella (Amorós, 2009).

De esta manera comenzó la Tercera ola del feminismo. Tras la Segunda Guerra Mundial la mujer seguía ocupando un papel fundamental dentro de la familia, principalmente como esposa, madre y ama de casa. Esto hacía que muchas de ellas no pudiesen desempeñar un trabajo remunerado fuera de casa, aunque no era este ni es el único ideal para la plenificación de la mujer como persona. De una manera similar lo explica la feminista estadounidense Betty Friedan (1921-2006). En su obra La Mística de la Feminidad pone nombre a lo que ella denomina "el problema que no tiene nombre" (Friedan, 2009), Friedan considera que la mujer es víctima de una heterodesignación o, dicho de otro modo, las mujeres eran víctimas de una designación de su identidad que les venía impuesta por la sociedad. La mujer debía asumir un rol y cumplir con unos estereotipos determinados, ya que, si se salía de ellos, sería socialmente rechazada. La mujer vivía, por tanto, insatisfecha cumpliendo ese papel en la sociedad puesto que sentían que estaban anteponiendo la voluntad o el cuidado de otros a sus propios deseos. Friedan fue cofundadora de la mayor organización feminista estadounidense, la Organización Nacional de Mujeres conocida como NOW (National Organization for Women), defensoras de un feminismo liberal y centradas en definir la situación de la mujer como de desigualdad y no como una explotación u opresión. Su principal cometido era el de luchar por conseguir cambios que conlleven una igualdad entre hombres y mujeres (Luengo González y Gutiérrez Esteban, 2011). Consideraban que la mujer había sido excluida de la vida pública y, por tanto, demandaban reformas que conllevasen la inclusión de la mujer en el mercado laboral.

Durante los años 60 las mujeres habían conseguido ya los derechos fundamentales y empezaron a ocupar puestos políticos y de poder. Sin embargo, la figura de la mujer dentro de las casas siguió jugando el mismo papel que en las décadas anteriores. En cierto modo, la mujer continuó asumiendo el rol de esposa, madre y ama de casa. Es en estos momentos, 
cuando apareció una nueva corriente de feminismo que pretendía acabar con esta situación, es el denominado como feminismo radical (Luengo González y Gutiérrez Esteban, 2011). Este movimiento pretendía acabar con los problemas de raíz, considerando que la base del problema era el patriarcado, según el cual nos encontramos en una sociedad en la que existe una dominación del hombre sobre la mujer. Los feministas radicales sostenían que este sistema de dominación se encontraba en todos los ámbitos (familiar, social, laboral, político, económico, etc.) y centraban la esencia del problema en el sistema de patriarcado, el género y la casta sexual. Para una mejor comprensión de las aspiraciones de este tipo de feminismo, a continuación, vamos a clarificar algunos de sus conceptos clave.

Se considera patriarcado al sistema en el que existe un predominio de autoridad del varón en la sociedad, específicamente sobre las mujeres y los niños. De esta manera, el patriarcado provoca que haya una distribución desigual en el reparto de derechos y oportunidades. Una de las aportaciones más importantes de este movimiento fue la creación de grupos de autoconciencia. Esta iniciativa perseguía que las mujeres más que apoyarse en ideologías previas, advirtiesen la opresión a la que estaban sometidas y, de esta manera, estuviesen motivadas para su lucha. Durante su actuación, no cesaron las marchas y manifestaciones, unidas a actos de sabotaje para poner de relieve la situación de opresión que estaba viviendo la mujer en esos momentos.

El feminismo radical y liberal han convivido durante varias décadas, sin embargo, es el feminismo radical el que ha alcanzado mayor protagonismo tanto durante la década de los sesenta como de los setenta. La principal diferencia entre uno y otro es que, mientras el feminismo liberal pretendía conseguir la igualdad de derechos entre hombres y mujeres, el feminismo radical iba más allá, buscando acabar con el sistema de patriarcado.

En la actualidad el tema de la mujer y el feminismo han pasado a estudiarse en muchos casos desde la perspectiva del género. Se ha creado una corriente de feminismo al que algunos denominan feminismo extremista (O'Leary, 2007). En muchas ocasiones se ha cambiado la batalla libre y abierta por una intromisión en las instituciones para lograr un impulso y conseguir una lucha más eficaz. Existe una corriente que se encarga de resaltar la opresión y la desigualdad que ha sufrido la mujer a lo largo de la historia. No obstante, esto a veces provoca que se pase por alto la labor de aquellas mujeres que sí consiguieron alcanzar una posición importante y, de esta manera, se impide resaltar sus logros. Asimismo, en numerosas ocasiones se omiten datos relevantes a lo largo de la historia de la humanidad, ofreciendo una versión sesgada y homogenizadora de la misma, cuando las 
diferencias entre las realidades son sustantivas. O no se toma en consideración que muchas mujeres no necesitan trabajar fuera de casa o realizar un trabajo remunerado para sentirse plenas $y$, en la actualidad, no se tiene apenas libertad para inclinarse por esta opción de vida. Pero, volviendo al tema que nos ocupa, los feministas de género o extremistas se intentaron apropiar del término feminismo y hacerlo propio, procurando que el feminismo implique o acapare la totalidad de sus ideales. El feminismo extremista sostiene que el hombre y la mujer deben tener los mismos derechos y la misma libertad, pero no se queda aquí, sostienen, además, que no existe diferencia de sexo entre hombres y mujeres, rechazando y obviando las diferencias biológicas, de ahí que se emplee el término género (gender) en lugar de sexo. Asimismo, niegan la importancia de la familia como unidad social y consideran que los gobiernos tienen que buscar la solución a los problemas. Otra nota característica de este movimiento es que persigue acabar con el concepto de familia, ya que considera que es una de las primeras causas de la opresión de la mujer. La mujer debe liberarse de esas ataduras que le impiden realizarse y conseguir sus objetivos. Las feministas extremistas creen que los hombres han construido la historia, la ciencia y la religión para oprimir a la mujer y que ellas deben hacer una nueva versión para alcanzar la liberación. Los sectores más radicales creen que la maternidad produce pensamiento de clases, que es el principal mal. Asimismo, consideran que el rol de madre y esposa es tedioso. En definitiva, la ideología imperante sostiene que existe un sistema de clases, en el que el hombre constituye la clase opresora, y la mujer la clase oprimida, por tanto, hay que abolir el sistema de clases aplicado a la diferencia sexual.

El siguiente punto a considerar es que, a partir de los años ochenta, el feminismo dio un paso más allá. Hasta ahora se había luchado por las mujeres en general, pero sin tomar conciencia de las diferencias específicas que existen, ya sean por cuestiones éticas, de raza, cultura, religión, etc. De este modo, como oposición al feminismo extremista o de género, aparecieron los defensores pro-familia o el feminismo de paridad, que se puede considerar un tipo más de feminismo, ya que defiende también los intereses y derechos de la mujer, pero con pensamientos e ideas contrarios a los planteados por los feministas extremistas. Quienes abogan por el hecho de que la mujer pueda hacer de la maternidad una vocación principal. Sostienen que el feminismo de género no busca una igualdad de derechos sino conseguir eliminar la condición de hombre o mujer para imponer la de género. Christina Hoff Sommers en su libro Who stole Feminism? (1995) (¿Quién robó el feminismo?) distingue entre "feminismo de género" y "feminismo de paridad". El feminismo de paridad ha sido un movimiento necesario en la historia, alabado por el mismo Juan Pablo II como feminismo 
auténtico y -como contrapunto- ha sido el feminismo de género o radical el que ha "robado" el feminismo hasta el punto de acabar incluso, con la idea de "mujer".

Por otra parte, también contamos en la actualidad con otra forma de feminismo: el ecofeminismo, que enlaza de alguna manera el feminismo con la ecología. Ante los problemas medioambientales que nos encontramos actualmente, ha surgido esta corriente de pensamiento que pretende alcanzar un horizonte utópico. Dentro del ecofeminismo encontramos varias corrientes y bastante enfrentadas. Françoise d'Eaubonne (1920-2005) publicó en 1974 un artículo en el que achacaba el problema de la sobrepoblación del planeta al sistema patriarcal, que impedía que las mujeres pudiesen decidir sobre su propio cuerpo. La alemana María Mies también encontró una relación entre la opresión en que viven las mujeres y el desarrollo de las sociedades industriales modernas, rechazando los recursos tecnológicos al considerarlos elementos de dominación del patriarcado. Una parte de este ecofeminismo trata de recuperar la naturaleza de la mujer, llamando a las mujeres a abandonar los estudios y sus trabajos y volver a consagrar su vida a procrear y al cuidado de sus hijos. Sin embargo, el pensamiento claro del ecofeminismo es abogar por la libertad de las mujeres en materia reproductiva.

Asimismo, una corriente más sería la que nos ofrece el transfeminismo (Navarro Pérez, 2019), estrechamente relacionado con el feminismo de género, ya que considera que el género es una construcción social y cultural que se utiliza como mecanismo de opresión a la mujer. Es un movimiento específico que se aplica a las personas transgénero o transexuales, pero el pensamiento es extensible a todas las mujeres en general. Tanto el feminismo como el transfeminismo sostienen que la mujer debe liberarse de los roles sociales impuestos, de modo que los transfeministas quieren ser valorados por sus actos y logros y no por el género que consideran que se les ha asignado al nacer.

En la actualidad también encontramos otro tipo de feminismo llamado feminismo de la igualdad con la idea de acabar con los roles de género. Sostiene que -los hombres y mujeres- al margen de su sexo o género son seres humanos de la misma manera. Con este fin, hay que cambiar el planteamiento actual sobre el sentido y la vida social de forma individual y colectiva. El feminismo de la igualdad se opone en esencia al feminismo de la diferencia. El feminismo de la diferencia hace una crítica a la búsqueda de la igualdad, ya que no niega la diferencia existente entre hombres y mujeres. El problema lo encontramos al considerar que lo contrario a igualdad es diferencia y no es así. Lo contrario a la igualdad es la desigualdad, por tanto, se deben tener presentes las diferencias entre hombres y mujeres, 
pero evitando que estas diferencias procuren un trato desigual o discriminatorio. En definitiva, no se puede perseguir que las mujeres sean iguales que los hombres porque acabarían con su propia esencia e identidad, lo que hay que conseguir es que la sociedad y la cultura respeten a ambos sexos.

Con un pensamiento más extremista surge el feminismo separatista que, como su propio nombre indica, busca la separación entre mujeres y hombres. Los defensores de esta corriente son conscientes de la diferencia entre hombres y mujeres y sostienen que la mujer debe mantenerse al margen de cualquier tipo de relación con los hombres. Derivado del feminismo separatista surge el feminismo lésbico que defiende las relaciones lésbicas como única alternativa para el correcto desarrollo de la mujer.

Por último, hacemos referencia al feminismo interseccional (Pereira, 2017), que parte de problemas sociales que afectan a multitud de grupos tradicionalmente discriminados, este feminismo tiene su raíz en combatir el machismo, el racismo, la xenofobia, la homofobia, la transfobia. Establece una relación entre el feminismo y la lucha contra todos estos problemas. El feminismo se convierte en el núcleo para iniciar un movimiento de lucha que sirva para acabar con las desigualdades que afectan a las mujeres derivado de todas esas circunstancias.

En definitiva, el feminismo, en los comienzos del movimiento se entendió como una lucha por conseguir una igualdad entre hombres y mujeres. En sus inicios se podía advertir una clara discriminación y opresión de la mujer en gran parte de los ámbitos de sus vidas, lo que evidencia que el movimiento feminista se sustentaba en unos ideales reales y perseguía unos fines objetivamente justos. Con el paso del tiempo, una vez que esas evidencias se fueron consiguiendo, empezaron a surgir otros propósitos ante los que ya no es tan sencillo posicionarse, ya que entre los propios movimientos feministas existen discrepancias sobre cuáles son los objetivos a perseguir. Ciertamente, lo más importante de todo, es que estos movimientos contribuyan a crear un mundo más justo e igualitario en cuanto a la defensa de los derechos y de la dignidad personal de la mujer. Por otro lado, es del todo necesario que, lo que en un principio se consideró un movimiento para acabar con las desigualdades y la discriminación, no se utilice en la actualidad precisamente para provocar lo que en sus inicios trataba de erradicar. 


\section{Conclusiones}

Recogiendo lo más importante de lo afirmado hasta aquí, sobre la historia del movimiento de liberación de la mujer, recordamos cómo en Grecia la mujer no jugó apenas ningún papel. Posteriormente, con el cristianismo, se introdujo una novedad gracias al respeto en el trato que mostró el mismo Cristo hacia las mujeres de su entorno. En la Edad Media destaca el poder de algunas abadesas y monjas de monasterios, que jugaron un papel relevante en la sociedad de su tiempo, al estar unidos entonces el poder religioso y el temporal. Por otra parte, en el Renacimiento nos encontramos con mujeres verdaderamente cultas, gracias y no sólo, por la posibilidad del acceso a la Universidad durante el siglo XVI. Pero este avance se vio frenado con la llegada del Siglo de las Luces, en el que las mujeres retornaron a la vida oculta, hasta que, ya en el XIX comenzaron a unirse, exigiendo su derecho al voto, en diversos movimientos feministas. Del siglo XX cabe destacar el impacto de las revoluciones sexuales, y del XXI, la problemática de la conciliación de la vida profesional y familiar, con la prioridad, en muchos casos, de la primera sobre la segunda.

Finalmente, ofrecimos un estudio detallado sobre el feminismo y sus diversas manifestaciones en la actualidad, en el que pusimos de manifiesto cómo el movimiento ha ido cambiando a lo largo de la historia. Estos cambios han ido adaptándose en consonancia con la situación de la mujer en cada momento. Hemos podido comprobar como gracias al feminismo se han alcanzado importantes logros para conseguir mejorar la situación de la mujer. Asimismo, se plantearon nuevas ramas del feminismo que persiguen objetivos muy diferentes, en algunos casos enfrentados y que, de no conseguir un equilibrio, pueden ensombrecer el verdadero fin del movimiento feminista. 


\section{Referencias}

Aguiar Barriga, N. (2020). "Una aproximación teórica a las olas del feminismo: la cuarta ola". Femeris vol. 5. no 2, 121-146.

Amorós, C. (2009). "Simone de Beauvoir: entre la vindicación y la crítica al androcentrismo". Investigaciones feministas, vol 0, 9-27.

Aparisi Miralles, Á. (2020). "La cuestión del género en Mayo del 68". En L. Jiménez, Mayo del 68 y su legado. Madrid: Fundación Universitaria Española.

Azcona, A. (2018). "La maternidad: una buena noticia". En S. Gallardo González, Mujer, familia y trabajo. Ávila: Universidad Católica de Ávila.

Bel Bravo, M. A. (1998). La mujer en la historia. Madrid: Encuentro.

Bel Bravo, M. A. (2017). "Mujer y sociedad en la Edad Moderna (siglos XV al XVIII)". En L. (. Jiménez, Mujer, isé lo que eres! Madrid: Fundación Universitaria Española.

Bel Bravo, M. A. (2020). La mujer en la historia: Ideología y realidad o cómo convertir el destino en oportunidad. Aula Magna, McGraw Hill Interamericana de España.

Biblia. (2010). Sagrada Biblia. Madrid: Biblioteca de Autores Cristianos.

Borges Morán, P. (1992). Historia de la Iglesia en Hispanoamérica y Filipinas, tomo I. Madrid: Biblioteca de Autores Cristianos.

Burgraff, J. (2007). Género (gender). En Lexicon. Términos ambiguos y discutidos sobre familia, vida y cuestiones éticas. Madrid: Palabra.

Cantera Montenegro, M. (2019). "Una fe que transforma el mundo". Versión electrónica, a partir del minuto 49. Ávila.

De Beauvoir, S. (2013). El segundo sexo. Madrid: Cátedra.

De Miguel, A. (s.f.). Los feminismos a través de la historia. En línea). Disponible en: https://web.ua.es/es/sedealicante/documentos/programa-de-actividades/2018-2019/losfeminismos-a-traves-de-la-historia.pdf [Consultado a 3 de septiembre de 2020]

Diccionario de la Real Academia Española. [Consultado a 3 de septiembre de 2020].

Friedan, B. (2009). La mística de la feminidad. Madrid: Cátedra.

Gallardo González, S. (2017). "La presencia de la mujer como "hogar" de lo humano". En L. Jiménez (ed.), Nuevas miradas sobre lo femenino. Madrid: Fundación Universitaria Española.

Gallardo Martín, A. I., y Sánchez Martínez, M. (2016). "El don de la paternidad y maternidad". En S. Gallardo González, La familia y sus retos. Ávila: Universidad Católica de Ávila.

Inaraja, M. S. (2012). "Entre el privilegio y la ciudadanía. Origen del marco de protección al indígena americano en la legislación virreinal". Fuego y Raya vol 2, no 4, 75-121.

Inaraja, M. S., y Expósito, J. A., (2015). Indigenismo y evangelización: la primera expansión del cristianismo en América. Madrid: Digital Reasons.

De Jesús, T. (2014). Libro de la Vida. Burgos: Monte Carmelo.

Jiménez, L. (2017). Mujer, isé lo que eres! Madrid: Fundación Universitaria Española.

Jiménez, L. (2020). Mayo del 68 y su legado. La universidad ante los retos del siglo XXI. Madrid: Fundación Universitaria Española.

Kuby, G. (2017). La Revolución Sexual Global. La destrucción de la libertad en nombre de la libertad.

Madrid: Didaskalos. 
Luengo González, M. R., y Gutiérrez Esteban, P. (2011). "Los feminismos en el siglo XXI: pluralidad de pensamientos". Brocar. Cuadernos de Investigación Histórica, no 35, 335-351.

Miyares, A. (1999). "El manifiesto de Seneca Falls". Leviatán: Revista de hechos e ideas no 75, 135158.

Navarro Pérez, P. (2019). "Transfeminismo y activismo queer: emergencia y cohabitación en las fronteras de la coalición". RECERCA. Revista De Pensament Y Anàlisi, 24 (2), 151-172.

O 'Learly, D. (2007). La agenda de género: redefiniendo la igualdad. San José: Promesa.

Pereira, I. (2017). "Interseccionalidad: El feminismo en la intersección de las luchas". Libre Pensamiento, (91), 27-33.

Ramos Gómez, M. (2017). "Mujer y trabajo: la mirada steiniana sobre una cuestión actual". En L. Jiménez, Nuevas miradas sobre lo femenino. Madrid: Fundación Universitaria Española.

Redondo Redondo, L. (2016). "Desenmascarar las ideologías". En S. Gallardo González, Persona e identidad. Ávila: Universidad Católica de Ávila.

Rodríguez Castellanos, M. F. (2020). "Desarrollo de la mujer en el ámbito musical a través del tiempo y breve historia del feminismo". MAGOTZI Boletín Científico De Artes Del IA, 8(16), 48-54. DOI: $10.29057 /$ ia.v8i16.4893

Sommers, C. H. (1995). Who stole feminism? How women have betrayed women. Nueva York: Simon and Schuster.

Spaemann, R. (1989). Lo natural y lo racional: ensayos de antropología. Madrid: Rialp.

\section{Cómo citar este trabajo}

Pro Velasco, M. L., \& Blázquez Gómez, R. (2021). El papel de la mujer como eje clave en la historia. Apuntes De Bioética, 4(2), 24-44. https://doi.org/10.35383/apuntes.v4i2.675

\section{Financiación}

El presente artículo no cuenta con financiación específica de agencias de financiamiento en los sectores público o privado para su desarrollo y/o publicación.

\section{Conflicto de interés}

El autor del artículo declara no tener ningún conflicto de intereses en su realización.

(c) Los autores. Este artículo es publicado por la Revista Apuntes de Bioética del Instituto de Bioética, Universidad Católica Santo Toribio de Mogrovejo.

Este es un artículo de acceso abierto, distribuido bajo los términos de la Licencia Creative Commons Atribución-NoComercial-CompartirIgual 4.0 Internacional (CC BY-NC-SA 4.0), que permite el uso no comercial, distribución y reproducción en cualquier medio, siempre que la obra original sea debidamente citada. 